\title{
Defining the impact of childhood adversities on cognitive deficits in psychosis: An exploratory analysis
}

\author{
Inga Schalinski ${ }^{\mathrm{a}, *}$, Martin H. Teicher ${ }^{\mathrm{b}, \mathrm{c}}$, Almut M. Carolus ${ }^{\mathrm{a}}$, Brigitte Rockstroh ${ }^{\mathrm{a}}$ \\ a Department of Psychology, University of Konstanz, Germany \\ b Department of Psychiatry, Harvard Medical School, Boston, MA, USA \\ c Developmental Biopsychiatry Research Program, McLean Hospital, Belmont, MA, USA
}

Keywords:

Sensitive period

Neurodevelopment

Childhood adversities

Cognitive functioning

\begin{abstract}
A B S T R A C T
Background: Exposure to adverse childhood experiences (ACE) and cognitive deficits are both prevalent in psy chosis. While it has been repeatedly demonstrated that ACE contribute to cognitive dysfunctions, the specific na ture of this contribution remains elusive. Recent evidence suggests that types of adversities during critical periods have deleterious effects on brain structures that are important for cognitive functioning. The present study sought to clarify which types of adversities experienced at which time during development aggravate cognitive deficits in psychosis.

Methods: Exposure to abuse and neglect during childhood and adolescence were retrospectively assessed in $N=$ 168 adult individuals with psychotic disorder. Conditioned random forest regression was used to define the im portance of type and timing of ACE for predicting domains of the MATRICS Consensus Cognitive Battery (MCCB). Results: Significant importance of ACE was determined for 5 out of 7 MCCB domains. Particularly abuse at age 3 contributed to dysfunctional cognitive domains attention, learning, and working memory. Social cognition was related to neglect experienced at 1112 years, and to cumulative ACE.

Conclusion: Abuse and neglect at periods when children spend substantial time in their families affect cognitive functioning, and hence aggravate dysfunction in psychosis. Results support the neurodevelopmental perspective on psychosis and the diagnostic value of type and timing of ACE.
\end{abstract}

\section{Introduction}

Exposure to adverse childhood experiences (ACE) and cognitive def icits are both prevalent in psychosis. With an attributional risk of 33\%, ACE are reliably related to psychosis (Varese et al., 2012) and have been integrated in etiological models (Read et al., 2014). In psychosis, cognitive deficits display a major source of disability (Nuechterlein et al., 2014), and individuals perform poorly on cognitive tasks with levels more than one standard deviation below those of controls (Carolus et al., 2014; Heinrichs, 2004).

Several studies report inverse associations of ACE and various cogni tive tasks (Lysaker et al., 2001; Schenkel et al., 2005; Shannon et al., 2011), also at early stages of psychosis (Aas et al., 2011; Campbell et al., 2013). Yet, inverse relationships between ACE and cognition were not confirmed in all studies (McCabe et al., 2012; Sideli et al., 2014), or were even found to be positive (Ruby et al., 2015).

Neurodevelopmental models offer the theoretical framework for un derstanding the link of ACE and cognitive functioning in general (Bick

\footnotetext{
* Corresponding author at: University of Konstanz, Department of Psychology, Box 905, 78457 Konstanz, Germany.

E-mail address: inga.schalinski@uni-konstanz.de (I. Schalinski).
}

and Nelson, 2016) and in psychosis (Catts et al., 2013; Feinberg, 1983; Keshavan et al., 2014). Brain systems enabling cognitive functions, in particular hippocampus and frontal cortex, follow different develop mental trajectories from infancy to early adolescence, and these periods are sensitive for environmental factors including ACE (Bick and Nelson, 2016; Pechtel and Pizzagalli, 2011; Teicher et al., 2016). Accordingly, cognitive dysfunction may be associated with ACE modified develop ment of these structures (Aas et al., 2013; Hoy et al., 2012; Catts et al., 2013; Ruby et al., 2014). Theoretical models based on normative brain development (Bick and Nelson, 2016) and on sensitive developmental periods (Teicher et al., 2016) suggest the hypothesis that within group variance in cognition can be partially explained by types and tim ings of ACE.

So far, studies focused on cumulative ACE (Shevlin et al., 2008), major abuse, particularly sexual abuse (Lysaker et al., 2001), or distinct age windows (e.g., ACE at 06 years of age in Hoy et al., 2012).

The present study evaluated more precisely which types and timings during development were important for cognitive domains that are typ ically impaired in schizophrenia according to the Measurement and Treatment Research in Schizophrenia (MATRICS). Together with the MATRICS Consensus Cognitive Battery (MCCB; Nuechterlein and Green, 2006), we used the Maltreatment and Abuse Chronology of 
Exposure (MACE) Scale (Teicher and Parigger, 2015) to retrieve retro spectively forms of abuse and neglect during childhood and adoles cence. The detailed information of exposure poses a statistical challenge of high collinearity in adjacent years as well as the large num ber of potential predictors. Data mining offers an adequate technique to overcome these obstacles and to identify important predictors (Breiman, 2001). Relationships of ACE and cognitive domains were ex pected along the maltreatment related alternations of brain circuits that are sensitive to type and timing of ACE and important for cognitions.

\section{Methods}

\subsection{Participants}

A total $N=168$ individuals with main diagnoses of psychotic spec trum disorder (World Health Organization, 1992) were recruited at the local center of psychiatry. Cognitive and ACE data of a subsample $(n=$ $62,36.9 \%$ ) were reported in a thematically different context (Carolus et al., 2014; Schalinski et al., 2015). Expert psychiatrists/psychotherapist made diagnosis upon admission: participants met criteria of a diagnosis of schizophrenia $76.2 \%$, schizoaffective disorder $10.7 \%$, and acute poly morphic psychotic disorder $13.1 \%$. Ninety five individuals with psycho sis were admitted for the first time (Table 1). We characterized the severity of psychopathology with the Positive and Negative Syndrome Scale (Kay et al., 1987). The majority of individuals with psychosis ( $n$ $=160$ ) was treated with neuroleptics for at least 2 weeks without change in dosage and type with a chlorpromazine equivalent dose of $M=534.5(S D=413.6)$, which is close to the commonly recommended maintenance dosage. For comparison purposes, $n=50$ non psychotic individuals with similar age and education were recruited from the community.

The study was reviewed and approved by the Institutional Review Board of the University of Konstanz. For all participants assessment took place in the post acute phase. The responsible psychologist/psychi atrists verified that the individual was in a sufficiently improved state to provide written informed consent and understand test and interview questions.

\subsection{Materials}

The MACE scale was developed to retrospectively capture the expo sure to ten forms of ACE between infancy and age 18, covering abuse (physical, verbal, non verbal emotional abuse, witnessing interparental abuse and abuse of siblings, peer related verbal abuse and physical bul lying, and intra , extra familial or peer related sexual abuse) and emo tional and physical neglect (Teicher and Parigger, 2015; Isele et al., 2014). For each of the 75 items (assigned to 10 subscales) experience was coded as yes no. For 'yes' responses the age of occurrence was eval uated in the same binary format for each year of life up to age 18. For each subscale, positively endorsed items were linearly interpolated to obtain severity scores that range from 0 to 10 . The overall severity of ACE was calculated using the sum of all 10 subscale severities (ranging from 0 to 100). The number of different forms (multiplicity) was oper ationalized as the number of those subscales that exceeded the defined cut off severity for clinically relevant exposure levels according to Isele et al. (2014). Similarly to the American version, the cut off scores are based on the raw values of positively endorsed items per subscale (Teicher and Parigger, 2015). The scores can be evaluated for each year (timing) and for each subscale (forms) and for cumulative mea sures (severity and multiplicity). ACE duration score summarizes the years of experience with a multiplicity score $\geq 1$ (ranging from 0 to 18). Forms of ACE were assigned to two types: abuse and neglect. The MACE scales demonstrate high quality psychometric properties: good convergent validity and an excellent retest reliability (Isele et al., 2014; Teicher and Parigger, 2015).

Cognitive performance was assessed with the MCCB ((Nuechterlein and Green, 2006), which covers the seven cognitive domains speed of processing, attention, working memory, verbal learning, visual learning, reasoning, and social cognition) with ten tests (Trail Making Test: Part A, Brief Assessment of cognition in Schizophrenia: Symbol Coding, Hop kins Verbal Learning Test Revised, Wechsler Memory Scale 3rd Ed.: Spatial Span, Letter Number Span, Neuropsychological Assessment Bat tery: Mazes, Brief Visual Memory Test Revised, Category Fluency: Ani mal Naming, Mayer Salovey Caruso Emotional Intelligence Test: Managing Emotions, Continuous Performance Test Identical Pairs). For evaluation of performance the raw scores are converted into age and gender corrected $\mathrm{T}$ scores based on data of the representative US

Table 1

Demographic and clinical data and adversity-related characteristics of individuals with psychosis $(N=168)$ and controls $(N=50)$.

\begin{tabular}{|c|c|c|c|}
\hline & Individuals with psychosis & Controls & Group comparison \\
\hline \multicolumn{4}{|l|}{ Demographic and clinical data } \\
\hline Age (in years) $M(S D)$ & $27.9(8.4)$ & $26.8(7.9)$ & $t(216)=0.83, p=0.407$ \\
\hline Female sex $n(\%)$ & $56(33.3 \%)$ & $22(44 \%)$ & $\chi_{(1)}^{2}=1.91, p=0.181$ \\
\hline First admission $n(\%)$ & $95(56.5 \%)$ & & \\
\hline Years of education $M(S D)$ & $11.7(1.7)$ & $11.4(1.37)$ & $t(216)=1.04, p=0.298$ \\
\hline PANSS sum score $M(S D)$ & $66(13.3)$ & & \\
\hline \multicolumn{4}{|l|}{ Childhood adversities } \\
\hline Duration $^{\mathrm{a}} M(S D)$ & $6.8(6.3)$ & $1.7(2.8)$ & $t(183.26)=8.03, p<0.001, d=1.04$ \\
\hline Multiplicity ${ }^{\mathrm{b}} M(S D)$ & $2.7(2.2)$ & $0.7(0.9)$ & $t(192.54)=9.17, p<0.001, d=1.19$ \\
\hline Severity ${ }^{c} M(S D)$ & $29.1(15.4)$ & $13.7(8.6)$ & $t(147.18)=9.06, p<0.001, d=1.23$ \\
\hline Participants with multiplicity $>0 n(\%)$ & $140(83.3 \%)$ & $22(44 \%)$ & $\chi_{(1)}^{2}=31.23, p<0.001, \phi=0.38$ \\
\hline \multicolumn{4}{|l|}{ МСCВ } \\
\hline Overall composite score & $37.00(10.17)$ & $49.32(8.41)$ & $t(216)=7.81, p<0.001, d=1.32$ \\
\hline Processing speed & $39.93(10.69)$ & $52.1(8.88)$ & $t(216)=7.32, p<0.001, d=1.24$ \\
\hline Attention & $36.34(9.44)$ & $43.66(9.37)$ & $t(216)=4.82, p<0.001, d=0.78$ \\
\hline Working memory & $44.22(10.19)$ & $51.34(8.38)$ & $t(216)=4.51, p<0.001, d=0.76$ \\
\hline Verbal learning & $46.45(9.94)$ & $52.36(10.63)$ & $t(216)=3.63, p<0.001, d=0.57$ \\
\hline Visual learning & $39.62(11.96)$ & $47.08(9.65)$ & $t(216)=4.03, p<0.001, d=0.69$ \\
\hline Reasoning and problem solving & $45.15(9.57)$ & $53.96(6.00)$ & $t(129.51)=7.83, p<0.001, d=1.10$ \\
\hline Social cognition & $42.26(10.83)$ & $47.34(8.46)$ & $t(216)=3.05, p=0.003, d=0.52$ \\
\hline
\end{tabular}

Note. PANSS = Positive and Negative Syndrome Scale. MCCB = MCCB = MATRICS Consensus Cognitive Battery. $d=$ Cohen's $d$.

a Years with a multiplicity score $\geq 1$ (ranging from 0 to 18).

b Number of different types (ranging from 0 to 10 ).

c Severity of childhood adversities (ranging from 0 to 100). 
community sample (Kern et al., 2008). The MCCB has demonstrated high quality psychometric properties including clinical importance for real world functioning (August et al., 2012).

\subsection{Analysis strategy}

Identifying important variables within a large number of predictors and high collinearity between predictors challenge traditional statistical approaches. To overcome these challenges, we applied two approaches in order to detect and select important predictors and converge findings of potentially important predictors: LASSO penalized (least absolute shrinkage and selection operator) regression and conditioned random forest regression. Both techniques are robust to small samples and large numbers of correlated predictors (Strobl et al., 2007; Tibshirani, 1996).

Analyses were conducted using $\mathrm{R}$ (version 3.0.2.) packages ('glmnet', 'party' and 'caret'). First, LASSO penalized regression was ap plied on all potential predictors (18 neglect and 18 abuse variables for each year between age 1 to 18), 3 cumulative ACE measures (duration, severity, multiplicity). Covariates were years of education, and two bi nary variables (first/repeated admission and gender) due to their po tential to influence with in group variation (Aas et al., 2014; Kern et al., 2008; Vaskinn et al., 2011). LASSO penalties shrink the $\beta$ estimates to zero in relation to the maximum likelihood estimates that could be caused by a high number of predictors and high collinearity and further acts as a selection operator (Tibshirani, 1996). Tuning parameters (using the lambda that gives the minimum for the mean cross validated error) determine the amount of shrinkage (zero means no shrinkage and therefore equalizes the maximum likelihood estimates, whereas an infinite value indicates infinite shrinkage and therefore setting the regression coefficients to zero). The LASSO penalized regression tends to select one representative of correlated predictors only and the output comprises $\beta$ estimates for those representative variables that were based on the leave group out cross validation of the LASSO penalty regression.

Second, conditioned random forest regression constitutes a machine learning strategy that detects important predictors from a large set of variables. This strategy has been applied previously on exposure data (Khan et al., 2015). The variant of Breiman's approach with conditional trees was chosen because no particular assumptions on distribution or descriptive characteristics are required and it is superior to other ap proaches in the analysis of complex data structures (Breiman, 2001; Strobl et al., 2009). The conditional grid is based on discretizing of the means of partition of the feature space of each individual tree and thus dealing with collinearity and avoiding that correlated predictors appear to be artificially more important. The algorithm has a built in val idation through splitting the data into a training (75\%) and holdout sample (25\%). This protects against overfitting and has an inherent pro cedure to estimate the permutation importance (see Strobl et al., 2009 for more detailed information). Furthermore, distribution free empiri cal probability scores were extracted based on 5000 permutation of each randomly reassigned predictor to estimate the likelihood of each predictor having occurred by chance. We selected only those predictors for post hoc explanatory analysis that verified importance according to LASSO penalized regression and conditioned random forest regression with differences of importance compared to permutated data on alpha $=0.050$ level (and alpha $<0.10$ for covariates). Selected predictors were entered in linear regression to establish the direction of relation ship. Furthermore, within group effects will be described using Cohen's d.

\section{Results}

\subsection{Childhood adversities and cognitive performance}

The majority of individuals with psychosis (83.3\%) reported the ex posure to at least one type of childhood adversity, whereas the prevalence in controls was considerably lower with $44 \%$. In general, in dividuals with psychosis reported a higher duration, more different forms (multiplicity) and more severe exposure to ACE compared to con trols (Table 1 ).

With an average MCCB overall T score of $M=37.00(S D=10.71$; Table 1) performance of the individuals with psychosis was lower com pared to non psychotic individuals $(M=49.32, S D=8.41)$. Effect sizes for group difference for the MCCB domains ranged from $d=0.52$ to $d=$ 1.24 .

\subsection{Modeling cognitive performance with ACE measures}

In psychosis, for five MCCB domains (attention, working memory, verbal and visual learning, social cognition) as well as MCCB overall score, measures of ACE exceeded the permutation based significant threshold in conditioned random forest regression and were selected as important predictors by LASSO penalized regression.

In particular, abuse at age 3 and age 12 verified variable importance for the MCCB overall score along with years of education and gender (Table 2). Both ACE measures were negatively associated with the MCCB overall score (Table 3), indicating lower scores consequent upon higher exposure to abuse at ages 3 and 12. Early abuse contributes to the within group variability ( $d=0.65$ ), and effect size between indi viduals with psychosis and controls (Table 2 ).

As illustrated in Fig. 1, abuse at age 3 was of maximum importance for attention, working memory, verbal and visual learning. Negative $\beta$ coefficients (ranging between -0.16 to -0.27 , Table 3 ) indicated the harmful effect of abuse at age 3 for theses domains. In addition attention was also related to neglect at age $6(\beta=0.15)$. Within psychosis the dif ferences for individuals with and without abuse at age 3 ranged from $d$ $=0.24$ for attention to $d=0.67$ for verbal learning. Years of education were positively associated with working memory and visual learning and male individuals with psychosis demonstrated lower scores in ver bal learning and working memory (Table 3 ).

Regarding social cognition, physical neglect at age 11 was of highest importance, in addition to the cumulative measures duration and mul tiplicity. Due to high collinearity between physical neglect and duration $(r>0.70)$, physical neglect at age 11 and multiplicity were included in the regression analyses. Both predictors showed a negative association with social cognition (Table 3 ). Using the median of neglect experiences at age 11 to distinguish individuals with low and high neglect experi ences resulted in a small effect size of $d=0.25$. None of the covariates verified importance.

\section{Discussion}

In line with previous reports (Heinrichs, 2004; Varese et al., 2012) individuals with psychosis demonstrated impaired cognitive perfor mance, and reported a longer duration, more different types, and higher severity of ACE compared to controls. The detailed assessment of ACE and their evaluation with conditioned random forest regression indicate a substantial impact of type and their timing during childhood on adult cognitive dysfunction. The present results highlight a negative impact of early abuse on basic cognitive functions such as attention and memory. Abuse, particularly at ages when children still spend substantial time in their families, co varies with fundamental cognitive abilities, thereby explaining lower performances in adult individuals with psychosis. The developmental period at age 1112 seems particularly vulnerable for neglect and impaired emotion regulation and mentalizing in adults with psychosis, which is embedded in generally higher exposure and longer duration of ACE. Small to moderate effect sizes show that ACE alone do not sufficiently explain cognitive deficits. Yet, they emphasize that both disease specific processes and adverse experiences contribute to cognitive dysfunctioning in psychosis.

In light of a neurodevelopmental perspective of psychosis (Catts et al., 2013) the present results may point to periods when cognitive 
Table 2

Results for those predictors that were selected by LASSO-penalized regression analysis and showed significance in conditioned random forest regression and group comparisons for the most important predictor (maximum importance in conditioned random forest regression).

\begin{tabular}{|c|c|c|c|c|c|c|c|}
\hline MCCB & Predictor & $\mathrm{B}_{\text {minMSE }}{ }^{\mathrm{a}}$ & $\begin{array}{l}\text { Random forest } M \\
(S D)\end{array}$ & $\begin{array}{l}\text { Permutation based } \\
p \text {-value }\end{array}$ & Effect size for & parisons & \\
\hline \multirow[t]{4}{*}{ Overall score } & Abuse sum age 3 & 0.90 & $2.53(0.16)$ & $p=0.0016$ & $\begin{array}{l}\mathrm{P}_{\text {Abuse } 3}<\mathrm{P}_{\mathrm{No}} \\
d=0.65^{\mathrm{b}}\end{array}$ & $\begin{array}{l}\mathrm{P}_{\mathrm{No}}<\mathrm{C} \\
d=1.20^{\mathrm{b}}\end{array}$ & $\begin{array}{l}\mathrm{P}_{\text {Abuse3 }}<\mathrm{C} \\
d=1.85^{\mathrm{b}}\end{array}$ \\
\hline & Abuse sum age 12 & 0.12 & $1.21(0.17)$ & $p=0.0286$ & & & \\
\hline & Years of education & 2.60 & $3.42(0.27)$ & $p=0.0024$ & & & \\
\hline & Gender ( $1=$ male, $2=$ female $)$ & 1.22 & $1.56(1.16)$ & $p=0.0286$ & & & \\
\hline \multirow[t]{3}{*}{ Attention } & & & & & $\mathrm{P}_{\text {Abuse } 3}<\mathrm{P}_{\mathrm{No}}$ & $\mathrm{P}_{\mathrm{No}_{\mathrm{o}}}<\mathrm{C}$ & $\mathrm{P}_{\text {Abuse3 } 3}<\mathrm{C}$ \\
\hline & Abuse sum age 3 & 0.77 & $2.25(0.26)$ & $p=0.00006$ & $d=0.24$ & $d=0.73$ & $d=0.95$ \\
\hline & Neglect sum age 6 & 0.06 & $0.76(0.06)$ & $p=0.0428$ & & & \\
\hline \multirow{5}{*}{$\begin{array}{l}\text { Working } \\
\text { memory }\end{array}$} & & & & & $\mathrm{P}_{\text {Abuse } 3}<\mathrm{P}_{\text {No }}$ & $\mathrm{P}_{\mathrm{No}}<\mathrm{C}$ & $\mathrm{P}_{\text {Abuse3 } 3}<\mathrm{C}$ \\
\hline & Abuse sum age 2 & 0.39 & $0.45(0.03)$ & $p=0.034$ & & & \\
\hline & Abuse sum age 3 & 0.04 & $1.31(0.09)$ & $p=0.0102$ & $d=0.57^{\mathrm{b}}$ & $d=0.63^{\mathrm{b}}$ & $d=1.19^{\mathrm{b}}$ \\
\hline & Years of education & 1.44 & $5.63(0.36)$ & $p=0.00008$ & & & \\
\hline & Gender ( $1=$ male, $2=$ female $)$ & 2.72 & $1.31(0.17)$ & $p=0.0326$ & & & \\
\hline \multirow[t]{3}{*}{ Verbal learning } & & & & & $\mathrm{P}_{\text {Abuse } 3}<\mathrm{P}_{\text {No }}$ & $\mathrm{P}_{\mathrm{No}}<\mathrm{C}$ & $\mathrm{P}_{\text {Abuse } 3}<\mathrm{C}$ \\
\hline & Abuse sum age 3 & 0.50 & $1.61(0.13)$ & $p=0.0036$ & $d=0.67^{c}$ & $d=0.43^{\mathrm{c}}$ & $d=1.10^{c}$ \\
\hline & Gender ( $1=$ male, $2=$ female $)$ & 2.20 & $0.98(0.12)$ & $p=0.0564$ & & & \\
\hline \multirow[t]{3}{*}{ Visual learning } & & & & & $\mathrm{P}_{\text {Abuse } 3}<\mathrm{P}_{\text {No }}$ & $\mathrm{P}_{\mathrm{No}}<\mathrm{C}$ & $\mathrm{P}_{\text {Abuse3 } 3}<\mathrm{C}$ \\
\hline & Abuse sum age 3 & 0.65 & $1.77(0.17)$ & $p=0.006$ & $d=0.51^{\mathrm{d}}$ & $d=0.58^{\mathrm{d}}$ & $d=1.10^{\mathrm{d}}$ \\
\hline & Years of education & 0.84 & $0.89(0.19)$ & $p=0.0654$ & & & \\
\hline \multirow[t]{5}{*}{ Social cognition } & & & & & $\mathrm{P}_{\text {HighNeglect11 }}<$ & $\mathrm{P}_{\text {LowNeglect11 }}<\mathrm{C}$ & $\mathrm{P}_{\text {HighNeglect11 }}<\mathrm{C}$ \\
\hline & Neglect sum age 11 & 0.84 & $2.71(0.34)$ & $p=0.00006$ & $\begin{array}{l}\mathrm{P}_{\text {LowNeglect }} \\
d=0.25\end{array}$ & $d=0.39$ & $d=0.67$ \\
\hline & Neglect sum age 12 & 0.0007 & $1.60(0.18)$ & $p=0.0038$ & & & \\
\hline & Duration $^{e}$ & 0.30 & $2.70(0.37)$ & $p=0.0036$ & & & \\
\hline & Multiplicity $^{\mathrm{f}}$ & 0.52 & $1.25(0.12)$ & $p=0.0238$ & & & \\
\hline
\end{tabular}

Note. $\mathrm{MCCB}=$ MATRICS Consensus Cognitive Battery. $\mathrm{P}=$ individuals with psychosis, $\mathrm{C}=$ control group.

a $\mathrm{B}_{\mathrm{minMSE}}=$ beta estimates based on the optimal lambda to find the minimum mean squared error in LASSO-penalized regression analysis.

b Adjusted for gender and years of education.

c Adjusted for gender.

d Adjusted for years of education.

e Years with a MACE multiplicity score $\geq 1$ (ranging from 0 to 18 ).

${ }^{\mathrm{f}}$ Number of different types (ranging from 0 to 10$) . d=$ Cohen's $d$.

Table 3

Summary of the regression analysis for measures of ACE and covariates predicting MCCB (A) overall score, (B) attention, (C) working memory, (D) verbal learning, (E) visual learning, and (F) social cognition.

\begin{tabular}{|c|c|c|c|c|c|}
\hline & Unstandardized coefficients $B$ & Unstandardized coefficients $S E$ & Standardized coefficient $\beta$ & $t$ & $p$ \\
\hline \multicolumn{6}{|c|}{ A. MCCB overall score $\left(R_{\text {adj }}^{2}=0.16, F_{(4163)}=9.12, p<0.001\right)$} \\
\hline Constant & 24.83 & 5.57 & & 4.46 & $<0.0001$ \\
\hline Sum score abuse 3 & 0.51 & 0.19 & 0.20 & 2.67 & 0.008 \\
\hline Sum score abuse 12 & 0.12 & 0.08 & 0.12 & 1.56 & 0.120 \\
\hline Gender $($ male $=1$, female $=0$ ) & 3.77 & 1.55 & 0.18 & 2.44 & 0.016 \\
\hline Years of education & 1.43 & 0.44 & 0.24 & 3.26 & 0.001 \\
\hline \multicolumn{6}{|c|}{ B. MCCB attention $\left(R_{\text {adj }}^{2}=0.07, F_{(2165)}=7.42, p=0.001\right)$} \\
\hline Constant & 36.00 & 0.94 & & 38.16 & $<0.0001$ \\
\hline Sum score abuse 3 & 0.65 & 0.18 & 0.27 & 3.61 & 0.0004 \\
\hline Sum score neglect 6 & 0.41 & 0.21 & 0.15 & 1.98 & 0.049 \\
\hline \multicolumn{6}{|c|}{ C. MCCB working memory $\left(R_{\text {adj }}^{2}=0.14, F_{(3164)}=10.23, p<0.0001\right)$} \\
\hline Constant & 27.57 & 5.56 & & 4.96 & $<0.0001$ \\
\hline Sum score abuse 3 & 0.4 & 0.19 & 0.16 & 2.16 & 0.033 \\
\hline Years of education & 1.69 & 0.45 & 0.28 & 3.80 & 0.0002 \\
\hline Gender $($ male $=1$, female $=0$ ) & 3.71 & 1.57 & 0.17 & 2.36 & 0.019 \\
\hline \multicolumn{6}{|c|}{ D. MCCB verbal learning $\left(R_{\text {adj }}^{2}=0.07, F_{(2165)}=6.01, p=0.003\right)$} \\
\hline Constant & 49.69 & 1.33 & & 37.45 & $<0.0001$ \\
\hline Sum score abuse 3 & 0.48 & 0.19 & 0.19 & 2.57 & 0.011 \\
\hline Gender $($ male $=1$, female $=0$ ) & 3.86 & 1.58 & 0.18 & 2.44 & 0.016 \\
\hline \multicolumn{6}{|c|}{ E. Visual learning $\left(R_{\text {adj }}^{2}=0.06, F_{(2165)}=6.52, p=0.002\right)$} \\
\hline Constant & 24.56 & 6.38 & & 3.85 & 0.0002 \\
\hline Sum score abuse 3 & 0.50 & 0.23 & 0.17 & 2.21 & 0.028 \\
\hline Years of education & 1.35 & 0.54 & 0.19 & 2.51 & 0.013 \\
\hline \multicolumn{6}{|c|}{ F. MCCB social cognition $\left(R_{\mathrm{adj}}^{2}=0.09, F_{(2165)}=9.03, p<0.0002\right)$} \\
\hline Constant & 46.17 & 1.27 & & 36.35 & $<0.0001$ \\
\hline Sum score neglect 11 & 0.68 & 0.28 & 0.23 & 2.45 & 0.015 \\
\hline MACE multiplicity & 0.60 & 0.45 & 0.12 & 1.35 & 0.179 \\
\hline
\end{tabular}

Note. $\mathrm{MCCB}=$ MATRICS Consensus Cognitive Battery. $\mathrm{ACE}=$ adverse childhood experiences. 

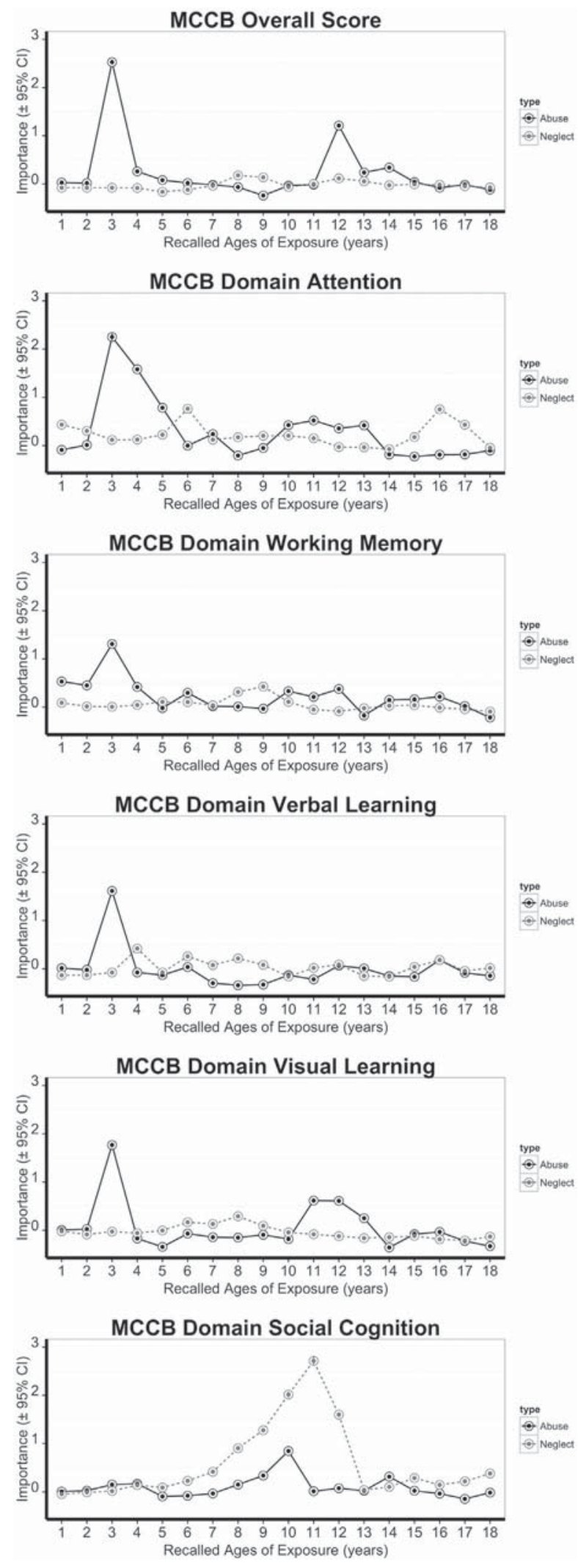

Fig. 1. Importance of measures of ACE for MCCB overall score and domains. Importance is represented per age (abscissa: years between 1 and 18) for abuse and neglect. Higher values indicate higher importance (independent of direction of the relationship). The error bars present the $95 \%$ confidence interval. ACE = adverse childhood experiences. MCCB $=$ MATRICS Consensus Cognitive Battery. functions develop and interact with ACE. This conclusion might be complemented by longitudinal studies in high risk populations in com parison to normative development. The profile of abuse and neglect in early childhood and preadolescence on cognitive functions overlaps with stress sensitive periods for hippocampus and frontal cortex devel opment (Andersen et al., 2008; Hoy et al., 2012; Teicher et al., 2016), and may go along with abnormalities of the hypothalamic pituitary ad renal axis (Ruby et al., 2014), and (epi)genetic factors (Aas et al., 2014). In line with the traumagenic neurodevelopmental model (Read et al., 2014), the present results support the assumption that abnormalities in psychosis are partly attributed to maltreatment related alternations. Furthermore, the differential contribution of early (intrafamilial) abuse on the development of fundamental cognitive abilities like attention and memory, and later (adolescent) neglect on higher cognitive func tions like social cognition can be understood in the concept of early sen sitive periods of hippocampal and later sensitive periods of prefrontal development (Bick and Nelson, 2016; Catts et al., 2013), and may reflect distinct effects as a function of type (McLaughlin et al., 2014; Vogel et al., 2011).

Limitations of the present study have to be noted. Retrospective as sessment of ACE prompted concerns regarding validity and reliability. Previous studies show sufficient test retest reliability in individuals with severe mental illness (Goodman et al., 1999), and show high con sistency in psychotic individuals (Fisher et al., 2011). While the MACE covers each year of age from birth to age 18 , limited and retrospectively unreliable information of exposure may be expected at very young age, prior to language fluency. Although very early abuse has been reported and is as deleterious as experiences at later age (Lupien et al., 2011), re liability of subjective reports and memories before age 3 may be questioned without reliable external sources. Furthermore, the small positive association of neglect and attention should be interpreted with caution since it only appeared in combination with abuse.

Effects of ACE on cognition have been reported also for other trau ma exposed groups (Sideli et al., 2014). Thus, the present results do not advocate a schizophrenia specific phenomenon. Moreover, the im pact of type and timing of ACE on psychopathology has been demon strated across diagnostic boundaries (Schalinski et al., 2016), while the specificity for cognitive dysfunctions in psychotic disorder need to be examined evaluated across diagnostic groups.

In conclusion, the delineation of type and timing advances our un derstanding of the contribution of ACE to adult cognitive deficits in schizophrenia. The findings support a neurodevelopmental model, al though the pathways and mechanisms translating the influence of ACE on cognition remain to be scrutinized. The results emphasize a par ticular role of abuse experienced during periods of early childhood and during adolescence. As the devastating impact of ACE on cognitive func tioning might also contribute to severity and course of illness, occupa tional and social outcomes, the specific characteristics of ACE should inform diagnostic routines (Schalinski et al., 2015) and may be an im portant measure in research on disease specific pathology.

\section{References}

Aas, M., Dazzan, P., Fisher, H.L., Morgan, C., Morgan, K., Reichenberg, A., Zanelli, J., Fearon, P., Jones, P.B., Murray, R.M., Pariante, C.M., 2011. Childhood trauma and cognitive function in first-episode affective and non-affective psychosis. Schizophr. Res. 129, $12-19$.

Aas, M., Haukvik, U.K., Djurovic, S., Bergmann, Ø., Athanasiu, L., Tesli, M.S., Hellvin, T., Agartz, I., Lorentzen, S., Sundet, K., 2013. BDNF val66met modulates the association between childhood trauma, cognitive and brain abnormalities in psychoses. Prog. Neuro-Psychopharmacol. Biol. Psychiatry 46, 181-188.

Aas, M., Dazzan, P., Mondelli, V., Melle, I., Murray, R.M., Pariante, C.M., 2014. A systematic review of cognitive function in first-episode psychosis, including a discussion on childhood trauma, stress, and inflammation. Front. Psychiatry 4, 182.

Andersen, S.L., Tomada, A., Vincow, E.S., Valente, E., Polcari, A., Teicher, M.H., 2008. Preliminary evidence for sensitive periods in the effect of childhood sexual abuse on regional brain development. J. Neuropsychiatr. Clin. Neurosci. 20, 292-301.

August, S.M., Kiwanuka, J.N., McMahon, R.P., Gold, J.M., 2012. The MATRICS consensus cognitive battery (MCCB): clinical and cognitive correlates. Schizophr. Res. 134, 76-82. 
Bick, J., Nelson, C.A., 2016. Early adverse experiences and the developing brain. Neuropsychopharmacology 41, 177-196.

Breiman, L., 2001. Random forests. Mach. Learn. 45, 5-32.

Campbell, C., Barrett, S., Shannon, C., Hoy, K., Rushe, T., Cooper, S., Mulholland, C., 2013. The relationship between childhood trauma and neuropsychological functioning in first episode psychosis. Psychosis 5, 48-59.

Carolus, A.M., Schubring, D., Popov, T.G., Popova, P., Miller, G.A., Rockstroh, B.S., 2014 Functional cognitive and cortical abnormalities in chronic and first-admission schizophrenia. Schizophr. Res. 157, 40-47.

Catts, V.S., Fung, S.J., Long, L.E., Joshi, D., Vercammen, A., Allen, K.M., Fillman, S.G. Rothmond, D.A., Sinclair, D., Tiwari, Y., 2013. Rethinking schizophrenia in the context of normal neurodevelopment. Front. Cell. Neurosci. 7, 60.

Feinberg, I., 1983. Schizophrenia: caused by a fault in programmed synaptic elimination during adolescence? J. Psychiatr. Res. 17, 319-334

Fisher, H.L., Craig, T.K., Fearon, P., Morgan, K., Dazzan, P., Lappin, J., Hutchinson, G., Doody, G.A., Jones, P.B., Murray, R.M., Morgan, C., 2011. Reliability and comparability of psychosis patients' retrospective reports of childhood abuse. Schizophr. Bull. 37, 546-553.

Goodman, L.A., Thompson, K.M., Weinfurt, K., Corl, S., Acker, P., Mueser, K.T., Rosenberg S.D., 1999. Reliability of reports of violent victimization and posttraumatic stress disorder among men and women with serious mental illness. J. Trauma. Stress. 12, 587-599.

Heinrichs, R.W., 2004. Meta-analysis and the science of schizophrenia: variant evidence or evidence of variants? Neurosci. Biobehav. Rev. 28, 379-394.

Hoy, K., Barrett, S., Shannon, C., Campbell, C., Watson, D., Rushe, T., Shevlin, M., Bai, F. Cooper, S., Mulholland, C., 2012. Childhood trauma and hippocampal and amygdalar volumes in first-episode psychosis. Schizophr. Bull. 38, 1162-1169.

Isele, D., Teicher, M.H., Ruf-Leuschner, M., Elbert, T., Kolassa, I.T., Schury, K., Schauer, M., 2014 KERF-ein Instrument zur umfassenden Ermittlung belastender Kindheitserfahrungen. [KERF-an instrument for measuring adverse childhood experiences: construction and psychometric evaluation of the German MACE (Maltreatment and Abuse Chronology of Exposure) scale]. Z. Klin. Psychol. Psychopathol. Psychther. 43, 121-130.

Kay, S.R., Flszbein, A., Opfer, L.A., 1987. The positive and negative syndrome scale (PANSS) for schizophrenia. Schizophr. Bull. 13, 261-276.

Kern, R.S., Nuechterlein, K.H., Green, M.F., Baade, L.E., Fenton, W.S., Gold, J.M., Keefe, R.S. Mesholam-Gately, R., Mintz, J., Seidmann, L.J., Stover, E., Marder, E.S., 2008. The MATRICS consensus cognitive battery, part 2: co-norming and standardization. Am. J. Psychiatry 165, 214-220.

Keshavan, M.S., Giedd, J., Lau, J.Y., Lewis, D.A., Paus, T., 2014. Changes in the adolescent brain and the pathophysiology of psychotic disorders. Lancet Psychiatry 1, 549-558.

Khan, A., McCormack, H.C., Bolger, E.A., McGreenery, C.E., Vitaliano, G., Polcari, A., Teicher M.H., 2015. Childhood maltreatment, depression, and suicidal ideation: critical importance of parental and peer emotional abuse during developmental sensitive periods in males and females. Front. Psychiatry 6, 42

Lupien, S.J., Parent, S., Evans, A.C., Tremblay, R.E., Zelazo, P.D., Corbo, V., Pruessner, J.C. Séguin, J.R., 2011. Larger amygdala but no change in hippocampal volume in 10year-old children exposed to maternal depressive symptomatology since birth. Proc. Natl. Acad. Sci. 108, 14324-14329.

Lysaker, P.H., Meyer, P., Evans, J.D., Marks, K.A., 2001. Neurocognitive and symptom correlates of self-reported childhood sexual abuse in schizophrenia spectrum disorders. Ann. Clin. Psychiatry 13, 89-92.

McCabe, K.L., Maloney, E.A., Stain, H.J., Loughland, C.M., Carr, V.J., 2012. Relationship between childhood adversity and clinical and cognitive features in schizophrenia. J. Psychiatr. Res. 46, 600-607.

McLaughlin, K.A., Sheridan, M.A., Lambert, H.K., 2014. Childhood adversity and neural development: deprivation and threat as distinct dimensions of early experience. Neurosci. Biobehav. Rev. 47, 578-591.

Nuechterlein, K.H., Green, M.F., 2006. MATRICS Consensus Cognitive Battery Manual. MATRICS Assessment Inc., Los Angeles, CA.
Nuechterlein, K.H., Ventura, J., Subotnik, K.L., Bartzokis, G., 2014. The early longitudinal course of cognitive deficits in schizophrenia. J. Clin. Psychiatry 75, 25-29.

Pechtel, P., Pizzagalli, D.A., 2011. Effects of early life stress on cognitive and affective function: an integrated review of human literature. Psychopharmacology 214, 55-70.

Read, J., Fosse, R., Moskowitz, A., Perry, B., 2014. The traumagenic neurodevelopmental model of psychosis revisited. Neuropsychiatry 4, 65-79.

Ruby, E., Polito, S., McMahon, K., Gorovitz, M., Corcoran, C., Malaspina, D., 2014. Pathways associating childhood trauma to the neurobiology of schizophrenia. Front. Psychol. Behav. Sci. 3, 1-17.

Ruby, E., Rothman, K., Corcoran, C., Goetz, R.R., Malaspina, D., 2015. Influence of early trauma on features of schizophrenia. Early Interv. Psychiatry. http://dx.doi.org/10. 1111/eip.12239.

Schalinski, I., Fischer, Y., Rockstroh, B., 2015. Impact of childhood adversities on the shortterm course of illness in psychotic spectrum disorders. Psychiatry Res. 228, 633-640.

Schalinski, I. Teicher, M.H. Nischk, D. Hinderer, E, Müller, O., Rockstroh, B. 2016. Type and timing of adverse childhood experiences differentially affect severity of PTSD, dissociative and depressive symptoms in adult inpatients. BMC Psychiatry 16, 295.

Schenkel, L.S., Spaulding, W.D., DiLillo, D., Silverstein, S.M., 2005. Histories of childhood maltreatment in schizophrenia: relationships with premorbid functioning, symptomatology, and cognitive deficits. Schizophr. Res. 76, 273-286.

Shannon, C., Douse, K., McCusker, C., Feeney, L., Barrett, S., Mulholland, C., 2011. The association between childhood trauma and memory functioning in schizophrenia. Schizophr. Bull. 37, 531-537.

Shevlin, M., Houston, J.E., Dorahy, M.J., Adamson, G., 2008. Cumulative traumas and psychosis: an analysis of the national comorbidity survey and the British Psychiatric Morbidity Survey. Schizophr. Bull. 34, 193-199.

Sideli, L., Fisher, H.L., Russo, M., Murray, R.M., Stilo, S.A., Wiffen, B.D.R., O'Connor, J.A., Falcone, M.A., Pintore, S.M., Ferraro, L., Mule, A., La Barbera, D., Morgan, C., Di Forti, M., 2014. Failure to find association between childhood abuse and cognition in first-episode psychosis patients. European Psychiatry 29, 32-35.

Strobl, C., Boulesteix, A.L., Zeileis, A., Hothorn, T., 2007. Bias in random forest variable importance measures: illustrations, sources and a solution. BMC Bioinformatics 8, 1 .

Strobl, C., Hothorn, T., Zeileis, A., 2009. Party on! Department of Statistics, Munich, Germany.

Teicher, M.H., Parigger, A., 2015. The 'Maltreatment and Abuse Chronology of Exposure'(MACE) scale for the retrospective assessment of abuse and neglect during development. PLoS One 10, e0117423.

Teicher, M.H., Samson, J.A., Anderson, C.M., Ohashi, K., 2016. The effects of childhood maltreatment on brain structure, function and connectivity. Nat. Rev. Neurosci. 17 652-666.

Tibshirani, R., 1996. Regression shrinkage and selection via the lasso. J. R. Stat. Soc. Ser. B Methodol. 267-288.

Varese, F., Smeets, F., Drukker, M., Lieverse, R, Lataster, T, Viechtbauer, W., Read, J., van Os, J., Bentall, R.P., 2012. Childhood adversities increase the risk of psychosis: a meta-analysis of patient-control, prospective-and cross-sectional cohort studies. Schizophr. Bull. 38, 661-671.

Vaskinn, A., Sundet, K., Simonsen, C., Hellvin, T., Melle, I., Andreassen, O.A., 2011. Sex differences in neuropsychological performance and social functioning in schizophrenia and bipolar disorder. Neuropsychology 25, 499-510.

Vogel, M., Meier, J., Grönke, S., Waage, M., Schneider, W., Freyberger, H.J., Klauer, T., 2011 Differential effects of childhood abuse and neglect: mediation by posttraumatic distress in neurotic disorder and negative symptoms in schizophrenia? Psychiatry Res. $189,121-127$.

World Health Organization, 1992. The ICD-10 Classification of Mental and Behavioural Disorders: Clinical Descriptions and Diagnostic Guidelines. World Health Organization, Geneva. 